\title{
Value of 3-dimensional speckle tracking echocardiography in the prediction of cardiovascular events in patients with hypertension complicated by acute myocardial infarction: a long-term follow-up study
}

\author{
Xiaoyan Chen ${ }^{1}$, Qingmei Yang ${ }^{1}$, Jianxiu Fang ${ }^{1}$, and Haifeng Guo ${ }^{1}$ \\ ${ }^{1}$ Affiliation not available
}

February 9, 2021

\begin{abstract}
Background Patients with hypertension complicated by acute myocardial infarction (AMI) have a poor prognosis. Identification of powerful predictors of recurring cardiovascular events (RCEs) is very important. This study sought to evaluate the predictive value of three-dimensional (3D) strain parameters for RCEs in patients with hypertension complicated by AMI. Methods We successfully followed up 62 patients with hypertension and AMI. Participants underwent three-dimensional echocardiography before, one week after, and one month after percutaneous coronary intervention (PCI). Left ventricular (LV) structural function parameters and three-dimensional strain parameters (3-dimensional global longitudinal strain (3D-GLS), 3-dimensional global circumferential strain (3D-GCS), 3-dimensional global radial strain (3D-GRS), and 3-dimensional global area strain (3D-GAS)) were acquired. We used a Cox model to determine the relationships between these parameters and RCEs. Results During follow-up (41.27 \pm 20.45 months), 20 patients (32.8\%) had RCEs, which were independently predicted one month after PCI by 3D-GLS (HR: 1.481, 95\%CI: 1.202-1.824) and 3D-GAS (HR: 1.254, 95\%CI: 1.093-1.440). The optimal 3D-GLS and 3DGAS cutoffs for predicting cardiac events were $>-12.5 \%$ [area under the receiver operating characteristic curve (AUC) 0.736 , 95\%CI 0.611-0.862, $\mathrm{P}=0.003$ )] and $>20.5 \%$ (AUC 0.685, 95\%CI 0.551-0.818, $\mathrm{P}=0.020$ ), respectively. Using logistic regression analysis, we constructed joint predictor $=(3 \mathrm{D}-\mathrm{GLS})+(3 \mathrm{D}-\mathrm{GAS}) \times 0.303 / 0.558$, and its cutoff point was $-22.36 \%$ (AUC 0.829 , 95\% CI 0.722-0.937, P<0.001). Conclusions 3D-GLS and 3D-GAS assessed one month after PCI can predict RCEs in patients with hypertension complicated by AMI. Additionally, the predicted value of (3D-GLS)+ (3D-GAS) $\times 0.303 / 0.558$ was higher than the predicted value of either parameter alone.
\end{abstract}

\section{Hosted file}

manuscript_-_final.pdf available at https://authorea.com/users/369642/articles/508165-valueof-3-dimensional-speckle-tracking-echocardiography-in-the-prediction-of-cardiovascularevents-in-patients-with-hypertension-complicated-by-acute-myocardial-infarction-a-longterm-follow-up-study 